\title{
Astronomy from the Antarctic Plateau: a global personal vision
}

\author{
Hans Zinnecker \\ Astrophysikalisches Institut Potsdam, An der Sternwarte 16, D-14482 Potsdam, Germany \\ email: hzinnecker@aip.de
}

\begin{abstract}
The Antarctic Plateau (Dome C, also Dome A) is emerging as an especially good sitefor astronomical observations (high, dry, cold, no wind, good free seeing above a certainboundary layer). Over the last few years, several meetings and conferences tookplace to discuss potential astrophysical science cases for such exceptional atmosphericconditions. I summarise my personal conclusions from these discussions and presenta global vision (roadmap) for Antarctic Astronomy for future optical, near-IR, thermal-IR, andfar-IR/sub-mm observations. The need for international collaboration between Europe, Australia and China is stressed.
\end{abstract}

Keywords. Antarctica, Telescopes, Site Testing, Instrumentation.

\section{Introduction}

The main advantages of the Antarctic Plateau $(\sim 3,200 \mathrm{~m})$ for astronomyare the weather conditions. At Dome $\mathrm{C}$ it is cold, high, dry and the sky quite stable. There are few clouds, hardly any wind and there are few aurorae. There is excellent night-time seeing in the Antarctic winter (May-August) above a ground layer of some $30 \mathrm{~m}$, with a median of about $\sim 0.3$ arcsec in thevisible. There are also 4 hours of excellent seeing on the ground duringday-time afternoon in the Antarctic summer (Nov-Feb), especially in thethermal infrared (remember the FWHM of the seeing scales with the inverse1/5 power of the wavelength) which allows us to make infrared astronomicalobservations in Antarctica in the summer-time, i.e. when the conditionsare much less harsh than in the winter time and without building towers (cf. Zinnecker (2007)). The Italian $80 \mathrm{~cm}$ IRAIT telescope will be the firstto prove the concept. Of course, optical observations require winter-timewhich in turn require telescopes on towers (except long-time seriesoptical photometric observations, where the seeing is not critical).

Although Dome A and Dome F may be slightly better astronomical sites (see the contributions by X. Cui from China and T. Ichikawa from Japan, respectively, in this Special Session and Proceedings), as a matter of fact Dome $\mathrm{C}$ and its infrastructure, i.e. the French-Italian Concordiastation, exist today and could be used (or even expanded) for optical/infrared/sub-mm astronomy, political problems notwithstanding.

\section{How best to exploit the Antarctica Plateau for astronomy now}

Astronomy in Antarctica is already going on at the Amundsen-Scott South Polestation. The South Pole $10 \mathrm{~m}$ radio/sub-mm telescope is installed andtaking data and the BICEP $25 \mathrm{~cm}$ aperture is studying CMB polarisationanisotropy (J. Carlstrom; Y.D. Takahashi, these Proc.). This raises the question whether Dome Cshould also have a big radio antenna, to carry out sub-mmobservations at 450 and 350 microns, and pushing for $\mathrm{THz}$ observationsin the 200 microns window. This would be a very good idea consideringthe 
very low precipitable water vapour (PWV) values, often less than $0.25 \mathrm{~mm}$. However, to be competitive a $25 \mathrm{~m}$ dish is needed (see the science case described by Minier (2008)) which wouldbe very expensive and would stretch the current logistics at theDome C Concordia station. In my view, this discourages going forsuch a Antarctic Submillimetre Telescope (AST) telescopeat the present time; furthermore, there is a major push for Dome Awhich is claimed to be an even better sub-mm site than Dome $\mathrm{C}$ andwhere our Chinese colleagues plan to build a $15 \mathrm{~m}$-class sub-mm-telescope. Finally, we should probably wait to see whether the $25 \mathrm{~m}$ CCAT (Cornell-Caltech Atacama Telescope, now under consideration) willbe realised on a very high site $(5,600 \mathrm{~m})$ near Cerro Chajnantor or not.

Leaving aside sub-mm telescopes, what should we do in optical and infrared astronomy? We have mentioned the advantages of the much reduced sky background in the thermal infrared regime $(3-5$ microns) compared to more temperate sites (factor $\sim 30$ ) due to the very cold atmosphere. There are several science cases for this part of the spectrum using a 2-3 m-class telescope (Burton (2005)), including molecular hydrogen surveysof the inner Galaxy, cool protostar and evolved star searchesin the LMC/SMC and also Galactic star forming regions in thesouthern hemisphere (e.g. Carina, Vela, etc.). These would allbenefit greatly from the increased spatial resolution with anAntarctic $2.5 \mathrm{~m}$ telescope, compared to Spitzer data obtained witha $85 \mathrm{~cm}$ telescope (factor 3 gain in angular resolution at 4 microns). Follow-up studies for NASA's soon-to-be-launched WISE $(40 \mathrm{~cm})$ near-to-thermal infrared all-sky survey telescope will also be an issue. It has been questioned whether all this would represent 'killer' science as opposed to fairly unique science. Perhaps the best use of a $2.5 \mathrm{~m}$ telescope (small enough to be affordable now but large enough to be a pathfinder for future large structures) may be to exploit the $\mathrm{K}_{\text {dark }}$ window (2.4 microns, Casali (2007)) wherethe reduced sky background allows us to really go deep (much below UKIDSS and VISTA) and carryout extra-galactic near-infrared surveys down to $\mathrm{K}_{\text {dark }}=25 \mathrm{mag}$. to study distant galaxy populations at high redshifts. JWST will provide competition, but only in principle and not in practice, as the FOV of JWST/NIRCAM is $3.4 \times 3.4$ arcmin. while an Antarctic survey telescope wouldhave $20 \times 20$ arcmin. or more, depending on the cost of IR-detectors.

\section{Roadmap for a Dome C Astronomical Observatory}

I consider a roadmap to be a temporal sequence of major milestones, with a beginning and an end. It should give realistic timescales to implement telescopes or instruments of increasing complexity. The beginning and first milestone would be to operate and obtain data with IRAIT as soon as possible (2010). The CAMISTIC bolometer cameraon IRAIT will probe the quality of the site for sub-mm/ THz (particularly for 200 microns) imaging observations. The second milestone should be an IR-optimised PLT-like telescope, if necessary with ground layeradaptive optics (first light in 5-10 years, 2015-2020). This should take advantage of the good Antarctic summer seeing andstart operating in the daytime, doing 0.3 arcsec $2.5-5$ microns imaging. At the same time, robotic optical telescopes like ICE-T could operatein the Antarctic winter, without towers (as the seeing is not criticalfor long-time series photometry). I would leave the lead for 2-4 m class optical telescope to our Chinese friends. The third milestone (in 10-15 years time) would be ALADDIN, the two-telescope thermal-IRinterferometer; it would then still be in time to act as an exo-zodiprecursor for DARWIN (the latter won't happen before 2025). The 4thmajor milestone (around 2030) could be a THz interferometer array (of the order of a dozen telescopes) complementing ALMA and SOFIA and being a pathfinder for a farIRinterferometer in space (ESA's future FIRI mission). The ultimateand 5th milestone 
that I see on the horizon (in the 2030-2040 timeframe) would be an optical/IR kilometric array of $2 \mathrm{~m}$ class telescopes.

\section{The need for international collaboration}

Astronomy in Antarctica is expensive for any country trying toestablish and maintain a permanent base on this remote continent. For example, the total operating costs of the Concordia station are 8 million Euro per year. Still, astronomy in Antarctica ismuch cheaper than ESA missions from space. Space projects are usually donein co-operation between several nations. Similarly, it would betoo costly if every nation would want to build their own suiteof telescopes in Antarctica. We have to share our resources. This means France and Italy will invite other European partners to participate in astronomy from Dome C. It also means that Dome C and Dome A should each concentrate and specialise on the best possible science doable from their respective sites. While Dome $\mathrm{C}$ might be best suited for near-infraredand thermal-infrared observations, Dome A (at 4,200 m) might be bestfor sub-mm and $\mathrm{THz}$ astronomy. Collaboration would reducecost and help to exchange scientific, technical, and logistic experience. These and other issues could be discussed at an IAU symposium on Antarctic Astronomy during the nextIAU General Assembly in 2012 in Beijing/China. By then, Chinese plans for Dome A will have firmed up, while Europe and Australia may have teamed up again at Dome Cpursuing their common interests for a PLT-like telescope. The first IRAIT measurements and results at around that time would hopefullyshow that Dome $\mathrm{C}$ lives up to its expectation as the premier astronomicalobserving site on the planet.

\section{Acknowledgements}

I would like to thank Michael Andersen and Mark McCaughreanfor critical discussions, as well as Michael Burton for hispatience with the manuscript. Travel support from the ARENAEC network the German DAAD is gratefully acknowledged.

\section{References}

Burton, M. G., Lawrence, J. S., Ashley, M. C. B., et al. 2005, PASA 22, 199

Casali, M. 2007, in: N. Epchtein and M. Candidi (eds.), $1^{\text {st }}$ ARENA Conference: Large Astronomical Infrastructures at Concordia, EAS Publications Series, vol. 25, p. 201

Minier, V. et al. 2008, in: H. Zinnecker, N. Epchtein \& H. Rauer (eds.), $2^{\text {nd }}$ ARENA Conference: The Astrophysical Science Cases at Dome C, EAS Publications Series, vol. 33, p. 21

Zinnecker, H., Andersen M. I., \& Correia, S. 2007, in: N. Epchtein and M. Candidi (eds.), $1^{s t}$ ARENA Conference: Large Astronomical Infrastructures at Concordia, EAS Publications Series, vol. 25, p. 183 